\title{
Extrahepatic Biliary Atresia
}

National Cancer Institute

\section{Source}

National Cancer Institute. Extrahepatic Biliary Atresia. NCI Thesaurus. Code C97069.

A cong enital disorder characterized by blockage or absence of the extrahepatic bile ducts. 